\title{
Diffusing magnetic Tb impurities and magnetotransport in strongly spin-polarized Bi films
}

\author{
Sergii Sologub, ${ }^{1}$ Daniel Lükermann, ${ }^{2}$ Herbert Pfnür, ${ }^{2}$ and Christoph Tegenkamp ${ }^{2, *}$ \\ ${ }^{1}$ Institute of Physics, National Academy of Sciences of Ukraine, Nauky Ave. 46, 03028 Kyiv, Ukraine \\ ${ }^{2}$ Institut für Festkörperphysik, Leibniz Universität Hannover, Appelstraße 2, 30167 Hannover, Germany
}

(Received 12 July 2013; published 6 September 2013)

\begin{abstract}
As a contribution to electronic transport within strongly spin-polarized surface states and its modification by adsorption of magnetic impurities we studied the adsorption of $\mathrm{Tb}$ (atomic magnetic moment $10 \mu_{B}$ ) on epitaxial $\mathrm{Bi}(111)$ films by means of surface sensitive (magneto)conductance and low-energy electron diffraction. Surface diffusion turned out to be non-negligible even at substrate temperatures of $10 \mathrm{~K}$. The $\mathrm{Tb}$ adatoms finally nucleate at intrinsic defects of the $\mathrm{Bi}(111)$ surface, where the $\mathrm{Tb}$ impurities act as dopants but not as scatterers. Nevertheless, time-dependent measurements allowed to determine also single-particle Tb scattering properties, as also supported by simulations of adsorption kinetics and time-dependent conductance. The magnetoconductance properties are characterized by small charge transfer $(0.05 e /$ atom $)$ and strong spin-orbit scattering, which in this case results only in strong reduction of the weak antilocalization effect but not a reversal to weak localization as for Fe and Co [see Lükermann, Sologub, Pfnür, Klein, Horn-von Hoegen, and Tegenkamp, Phys. Rev. B 86, 195432 (2012)]. Although Tb has a magnetic moment, which is by far higher than for adsorbed Fe and Co, it turns out that the $f$ electrons of Tb play essentially no role in scattering of the conduction electrons, yielding an even smaller scattering cross section than that for Fe and Co. The adatom coordination (interstitial or on the surface) may also play an important role.
\end{abstract}

DOI: 10.1103/PhysRevB.88.115412

PACS number(s): 73.40.-c, 61.05.jh, 78.40.Kc

\section{INTRODUCTION}

Fundamental aspects of spin-polarized currents are relevant for various magnetoelectrical concepts, ${ }^{1,2}$ and have been successfully mapped onto surface structures. Systems with strong spin-orbit coupling (SOC) reveal inherently spin-polarized surface states due to the broken space-inversion symmetry. Among others, Bi turned out to be a valuable prototype system to study the interplay between the electronic band structure and propagating electrons in these surface states. ${ }^{3-9}$ In this respect, epitaxial $\mathrm{Bi}(111)$ films were shown to be attractive study objects, since the bulk states become gapped due to robust confinement. Thus the surface states can be probed directly by transport measurements. ${ }^{7,10}$

The surfaces of such semimetallic thin-film systems exhibit strong similarities with topological insulators (TI). Therefore they can be used to study related questions without dealing with stoichiometry problems or adsorbate-induced gaps of the topological surface states. ${ }^{11}$ A central topic is the scattering of electrons within these spin-polarized surface states because time-reversal symmetry (TRS) requires a suppression of direct backscattering of charge carriers between states with opposite wave vectors and spin orientations. The suppression of backscattering was shown recently for Bi thin films ${ }^{6}$ and $\mathrm{Bi}_{1-x} \mathrm{Sb}_{x}{ }^{12}$ by the analysis of the quasiparticle interference (QPI) patterns. On the other hand, scatterers with strong spin-orbit coupling allow to change both spin and momentum so that this rule may be violated.

A main source for scattering of electrons are defects and disorder in these systems. For impurities carrying a net magnetic moment, the orthogonality between initial and final state is lifted by breaking (locally) TRS, as seen, e.g., for Fe impurities on $\mathrm{Bi}_{2} \mathrm{Se}_{3} \cdot{ }^{13}$ For $\mathrm{Bi}(111)$ this issue has been addressed by studying the interference patterns of the $\operatorname{Bi}(111)$ surface states around various defects. ${ }^{6,9}$ Recently, we have characterized the magnetic and nonmagnetic impurities in magnetoconductance by analyzing the effect of weak (anti)localization. ${ }^{14}$ These studies have shown that a straightforward argumentation in terms of TRS is rather limited, since additional effects such as charge transfer and hybridization have to be considered. In our present study, we will add surface diffusion of the dopants to this list.

As mentioned, the break of TRS is an idealized model, which does not take into account chemical bonds and hybridization. This means that details of the energetic alignment of the magnetic orbital as well as their spatial distribution under consideration of the participating valence shells and adsorption site are usually not considered. The importance of these modifications became already obvious from recent ARPES measurements performed on TIs, where, depending on the adsorption site, different types of doping have been observed. ${ }^{15}$ In a recent STM study supplemented by DFT, it was nicely demonstrated that the coordination and, thus, the oxidation state of the impurity atom triggers the doping level and residual magnetic moment. ${ }^{13} \mathrm{For} \mathrm{Fe} / \mathrm{Bi}_{2} \mathrm{Se}_{3}$, the position of the $\mathrm{Fe}$ impurity can be changed from an on-top position into a substitutional defect site position simply by thermal annealing, associated with an increase of the magnetic moment. Nevertheless, the impact of an impurity on the (local) band structure of the host as well as the detailed process of spin excitation upon scattering remain unclear at moment.

In the study presented here, the effects of diffusion, charge transfer, and magnetic moment dependent scattering at impurities adsorbed on epitaxial $\mathrm{Bi}(111)$ films were analyzed by means of temperature-dependent dc and magnetotransport measurements. Terbium ( $\mathrm{Tb}$ ) was chosen because as a rareearth atom it is expected to posses a large magnetic momentum. Upon adsorption of $\mathrm{Tb}$ the $4 f$ electrons remain localized and only $6 s$ and $5 d$ shells contribute to bonding and doping between the rare-earth adsorbate and the substrate. Therefore its extraordinarily high magnetic moment of almost $10 \mu_{B}{ }^{16}$ 
is expected to survive adsorption. Since diffusion is not negligible even at a surface temperature of $10 \mathrm{~K}$, as we will show, $\mathrm{Tb}$ must remain on the $\mathrm{Bi}(111)$-surface, in contrast to $\mathrm{Fe}$ and $\mathrm{Co}$, investigated in detail in a former study. ${ }^{14}$ The importance of diffusion is supported by simulations based on simple nucleation theory.

Our paper is organized as follows. First, we will present data obtained with time dependent measurement of structure, obtained with low-energy electron diffraction (LEED), and corresponding dc conductance measurements before we proceed with magnetoconductance and Hall measurements. Finally, we present a kinetic model that describes the observed time dependent conduction data semiquantitatively and end with a discussion of our results.

\section{EXPERIMENTAL SETUP}

Low-doped $\mathrm{Si}(111)$ samples $(\rho>1000 \Omega \mathrm{cm})$ have been used as substrates for the film growth and subsequent conductivity measurements. All experiments have been performed in ultrahigh vacuum. Details about the fabrication of the contacts and the in situ cleaning procedures of the Si samples are described elsewhere. ${ }^{7,17} \mathrm{Bi}$ has been evaporated out of a ceramic crucible, while $\mathrm{Tb}$ has been evaporated by direct current heating of a tantalum stripe to which a small piece of pure $\mathrm{Tb}$ has been attached by spot welding. The quality and crystallinity of the Si surface and Bi films have been checked by low-energy electron diffraction (LEED). Monocrystalline films with average grain sizes in the order of $10 \mathrm{~nm}$ are

(a)
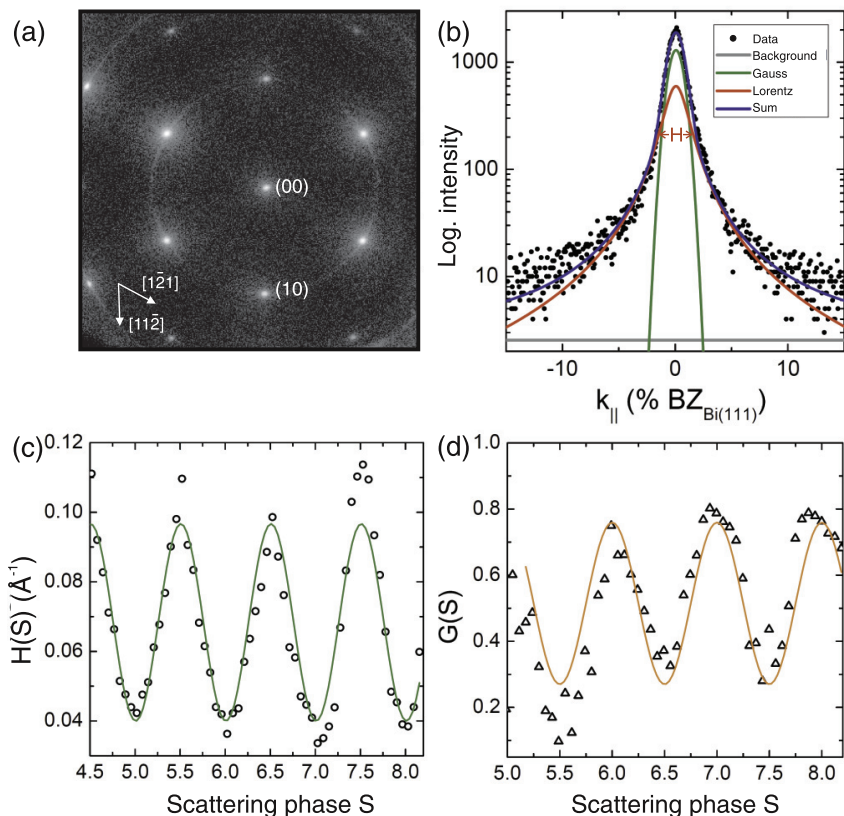

FIG. 1. (Color online) (a) Diffraction pattern of a 20-BL Bi film grown epitaxially on $\operatorname{Si}(111) 7 \times 7$. The primary electron energy is $E=96 \mathrm{eV}$ and close to an out of phase scattering condition, step height of $\mathrm{Bi}(111)$ is $d_{\mathrm{Bi}(111)}=3.94 \AA$. (b) Spot profile of the (00) spot showing the contributions of the central peak and a Lorentzian shoulder. (c) Variation of the FWHM of the Lorentzian [ $H(S)$ curve]. (d) Relative intensity of the Gaussian of the (00) peak versus the scattering phase $S[G(S)$ curve]. For details see text. routinely obtained by adsorption of $\mathrm{Bi}$ at $200 \mathrm{~K}$ followed by annealing to $450 \mathrm{~K}$ for several minutes. ${ }^{7,14}$ Figure 1(a) shows a typical LEED-pattern of a 20 bilayer $(\mathrm{BL}, 1 \mathrm{BL}=1.14 \times$ $10^{15}$ atoms $/ \mathrm{cm}^{2}$ ) thick $\mathrm{Bi}(111)$ film (here we use the rhombohedral notation for indexing the Bi planes, ${ }^{18}$ i.e., the surface normal coincides with the [111] direction). The faint ring structure is to a small rotational disorder of the (111)-textured domains.

The amount of $\mathrm{Tb}$ deposited onto clean $\mathrm{Bi}(111)$ films has been calibrated using the minimum in conductance during deposition expected to occur at $0.5 \mathrm{ML}$ (1 ML is defined as $0.5 \mathrm{BL}$, i.e., the number of $\mathrm{Bi}$ atoms in the top of the $\mathrm{Bi}$ bilayer). The calibration is supported by LEED measurements showing a complete suppression of the Bi diffraction spots after the adsorption of a complete but disordered $\mathrm{Tb}$ monolayer at low temperatures. The deposited $\mathrm{Tb}$ concentration has been further calibrated by the $\sqrt{3} \times \sqrt{3}$ reconstruction at 0.33 ML found both on $\mathrm{Si}(111)$ and $\mathrm{Bi}(111)$ films, and the global minimum in the conductance at $0.5 \mathrm{ML}$, respectively.

For magnetotransport measurements the prepared Bi films were transferred in situ to where the magnet $(B= \pm 4 \mathrm{~T})$ is located. The adsorption experiments were performed at various temperatures whereas the magnetoconductance measurements were performed at $10 \mathrm{~K}$ only to minimize contributions from inelastic scattering.

\section{RESULTS AND DISCUSSION}

\section{A. Structure and roughness of pristine $\mathrm{Bi}(\mathbf{1 1 1})$ films}

In a previous study, we showed that at temperatures below $60 \mathrm{~K}$ the electronic transport is dominated strongly by conduction in the surface states. ${ }^{7}$ Therefore electronic transport is limited by scattering at surface defects in this temperature range. This is the reason why we describe here a detailed analysis of the surface morphology of the Bi films. We analyzed both the step density and the surface roughness in detail by spot profile analysis in low-energy electron diffraction (SPA-LEED). As mentioned above the growth of $\mathrm{Bi}$ on $\mathrm{Si}(111)$ at $200 \mathrm{~K}$ followed by annealing to $450-\mathrm{K}$ results in the formation of (111)-textured single domain Bi films. A LEED pattern of a 20-BL-thick Bi film is shown in Fig. 1(a).

More details about the roughness are gathered from the analysis of diffraction profiles, e.g., shown for the specular spot in Fig. 1(b). The profiles taken at different electron energies are well described by a central Gaussian peak and a shoulder (the Lorentzian function). The latter is sensitive to the lateral roughness, and its full width at half maximum (FWHM) reaches its maximum value at electron energies which correspond to out-of-phase scattering conditions. The result of fitted FWHMs is shown in Fig. 1(c) for a broad range of electronic scattering phases $S\left(S:=\frac{k_{\perp} d}{2 \pi}\right)$ between adjacent terraces, assuming a step height $\mathrm{d}$ of one bilayer. This assumption is well justified, since the minima in FWHM always occur at integers of $2 \pi$ [see Fig. 1(c)]. The oscillatory behavior is well described by $4[1-\cos (2 \pi S)] / \Gamma$, where $\Gamma$ denotes the average terrace length (green curve in this figure). The evaluation within this kinematic approximation ${ }^{19}$ yields an average terrace length of $14 \mathrm{~nm}$. The vertical roughness of the film can be quantified by analyzing the ratio, $G(S)$, 


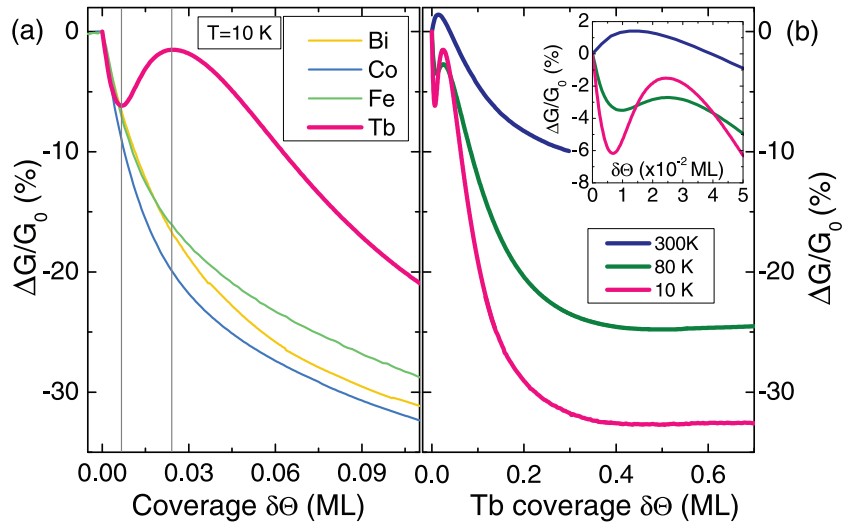

FIG. 2. (Color online) Relative change of the surface conductance during deposition of $\mathrm{Tb}$ on 20-BL Bi. The flux was $2 \times 10^{-5} \mathrm{ML} / \mathrm{s}$. (a) Comparison between the adsorption of $\mathrm{Tb}$ to the cases of $\mathrm{Bi}, \mathrm{Fe}$, and Co adsorption (data taken from Lükermann et al. ${ }^{14}$ ) at $T=10 \mathrm{~K}$. Only in case of $\mathrm{Tb}$ adsorption a recovery of conductance is found. (b) Relative change of the surface conductance as a function of $\mathrm{Tb}$ coverage for various temperatures. The inset shows a close up for better visibility. Interestingly, at $300 \mathrm{~K}$ the conductance with $0.01 \mathrm{ML}$ $\mathrm{Tb}$ is higher compared to the uncovered surface. For further details see text.

of the integral intensity of the central peak divided by the total intensity of the diffraction peak $\left(I_{\text {peak }}+I_{\text {shoulder }}\right)$ for various scattering conditions, as plotted in Fig. 1(d). The modulation of $G(S)$ is nicely described by a model which assumes only two step heights and uncorrelated steps: $G(S)=$ $p_{0}^{2}+p_{1}^{2}+2 p_{0} p_{1} \cos (2 \pi S)$, where the $p_{i}$ 's denote the fraction of the visible surface atoms of the $i$ 's layer. In other words, the $\mathrm{Bi}(111)$ films are extremely smooth and fully percolated. Furthermore, around the in-phase scatting conditions, the data can be approximated by $G(S) \approx \exp \left[-\Delta^{2}(2 \pi \delta S)^{2}\right]$ (see Ref. 20) with $\delta$ S denoting the deviation of the scattering phase $S$ from the next integer value. $\Delta$ is the root mean square (rms) value of the vertical roughness. We obtain a value for $\delta \mathrm{S}$ of $1.4 \AA$ for our epitaxial Bi(111) films.

\section{B. Conductance changes induced by $\mathbf{T b}$ adsorption}

The impact of atomic sized scatterers and the importance of diffusion, nucleation and charge transfer can be comprehensively studied by means of surface sensitive transport in combination with low-energy electron diffraction. The changes of the conductance upon adsorption of four different adsorbates is shown in Fig. 2. The fluxes were as low as $2 \times 10^{-5} \mathrm{ML} / \mathrm{s}$ and adsorption was performed at a substrate temperature of $10 \mathrm{~K}$. Contributions from bulk states are negligible at these temperatures, thus the electron transport is mainly governed by $\mathrm{Bi}(111)$ surface states. ${ }^{7}$ Figure 2 (a) shows the relative changes of the conductances during adsorption of $\mathrm{Bi}, \mathrm{Co}, \mathrm{Fe}$, and $\mathrm{Tb}$. While the conductance decreases gradually for the three former adsorbates, $\mathrm{Tb}$ shows a completely different behavior. Initially the conductance value decreases with increasing $\mathrm{Tb}$ excess coverage similar to the other three adsorbates. However, the conductance recovers around $0.025 \mathrm{ML}$ leading clearly to a maximum in the conductance curve. The monotonous decrease of the conductance upon adsorption of the other elements shown in Fig. 2(a) as well as the differences among each other have been explained in terms of random adsorption, coupled with significant charge transfer from Bi to the metal atoms in the case of $\mathrm{Fe}$ and Co. Details are reported in Ref. 14.

The appearance of the recovery maximum upon adsorption of $\mathrm{Tb}$ can be tuned by changing the deposition rate and substrate temperature. In this study, we restrict ourselves to the variation of substrate temperature as stable rates and low pressures during evaporation $\left(p<2 \times 10^{-8} \mathrm{~Pa}\right)$ were obtained only in the low-flux regime. The effect is demonstrated in Fig. 2(b) exemplarily for three different substrate temperatures. The inset shows a magnification of the low-coverage regime: compared to the $10 \mathrm{~K}$ measurements, the $\Delta G / G_{0}$ curve at $80 \mathrm{~K}$ shows a maximum at almost the same position, but the maximum is less pronounced and the overall decrease of conductance is smaller. The latter is a direct consequence of bulk state contributions to conductance above $60 \mathrm{~K}^{7}$ so that surface sensitivity is reduced in our transport experiments at this temperature, and even more at $300 \mathrm{~K}$, where the maximum
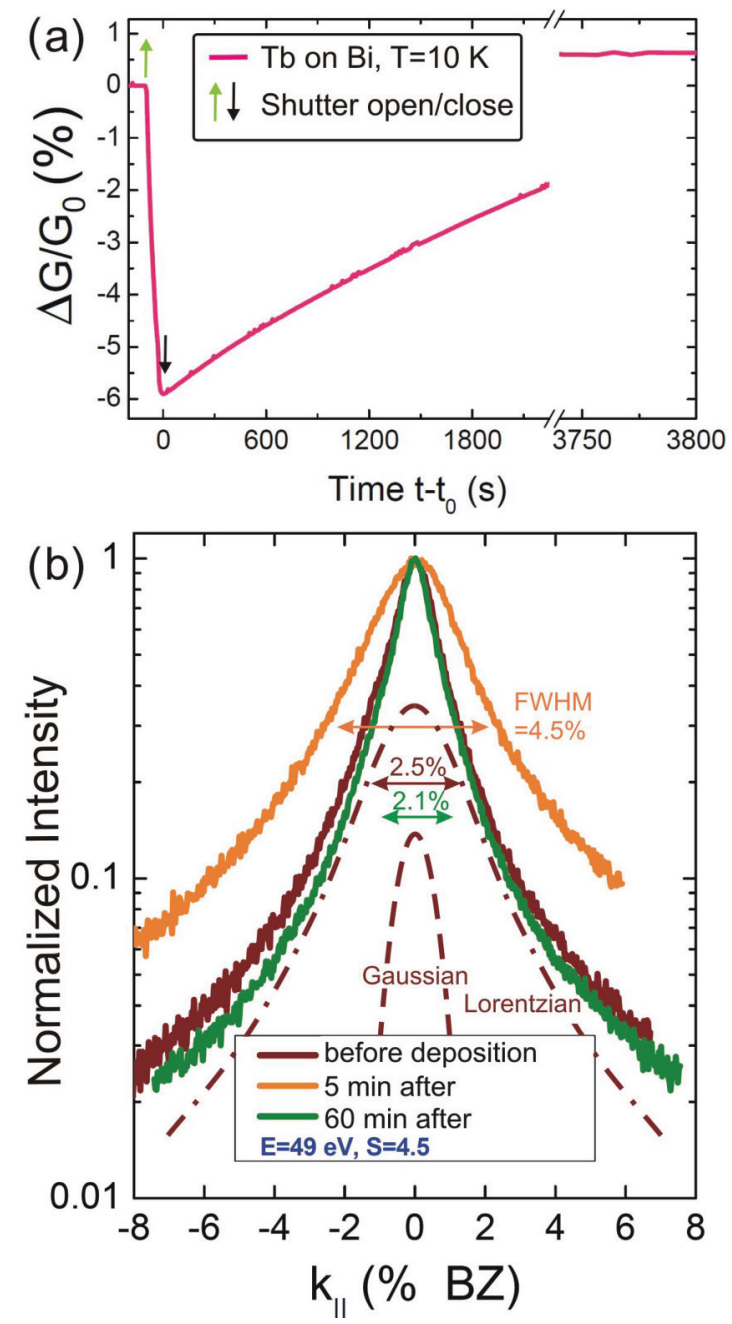

FIG. 3. (Color online) Change of the relative conductance (a) and the intensity of the (00)-diffraction spot (b) after adsorption of $0.005 \mathrm{ML} \mathrm{Tb}$ at $10 \mathrm{~K}$. The diffraction spots were taken at an out-of phase scattering condition $(49 \mathrm{eV})$. After closing the shutter of the evaporator both quantities recover fully by time. 
has shifted also to lower excess coverages. This already shows that surface diffusion is important for Te adsorption as also shown by our simulations presented below. Most interestingly, at $300 \mathrm{~K}$ initial adsorption of $\mathrm{Tb}$ only results in an increase of conductance, i.e., to a value above that at zero coverage. Apparently, the scattering processes are overcompensated by charge transfer processes, similarly to those found for $\mathrm{Fe}$ and $\mathrm{Co}$ adsorption on $\mathrm{Bi}(111)$ films. ${ }^{14}$ At lower substrate temperatures, the effect of doping is not directly obvious from $G(\delta \Theta)$ measurements. Further details are discussed in context with the magnetotransport data presented below.

\section{Time-resolved recovery process of $\mathbf{T b}$ adsorption}

In order to illustrate that indeed, even at $10 \mathrm{~K}$, surface diffusion is still happening, we recorded structural changes in addition to conductance. Figure 3 shows the time evolution of both quantities after deposition $0.005 \mathrm{ML}$ at $10 \mathrm{~K}$. To start with transport, Figure 3(a) shows the recovery process right after closing the shutter. As can be seen, the conductance gradually increases and reaches its initial value on the time scale of one hour. Waiting even longer, conductance was again found to be larger than the initial value by $0.7 \%$, which we attribute to doping by the adatoms, as discussed in context with Fig. 2(b) for the $300 \mathrm{~K}$ measurement.

The relevance of surface diffusion is nicely supported by SPA-LEED measurements. The evolution of the specular spot measured at an out-of-phase condition is shown in Fig. 3(b). Immediately after adsorption of $0.005 \mathrm{ML}$ of $\mathrm{Tb}$ the full width of half maximum (FWHM) and the background both increase by a factor of two. Interestingly, after one hour, the spot profile of the (00) spot is identical (or even slightly smaller) than initially without $\mathrm{Tb}$, i.e., the defect concentration after adsorption is essentially the same as before.

This behavior can be well understood. Random adsorption of $\mathrm{Tb}$ at the beginning of this experiment results in an average distance of 15 lattice constants $a$. Since we measure at an out-of-phase condition, we are most sensitive to disorder. Furthermore, $\mathrm{Tb}$ is a strong scatterer so that the spot profile is governed by scattering at $\mathrm{Tb}$ atoms, which are randomly distributed. The scattering factor of $\mathrm{Tb}$ may be further enhanced by local relaxation of $\mathrm{Bi}$ atoms due to chemical bonding. The profile width after 5 min corresponds to an average distance of $22 a$. It suggests that part of the Tb atoms have already been condensed to islands or been trapped at step edges. In any case, this requires diffusion over many lattice constants already at $10 \mathrm{~K}$. Trapping at step edges seems to be indeed the dominant mechanism here, since the original profile is recovered. This whole annealing process is only conceivable if $\mathrm{Tb}$ atoms are adsorbed just on the surface, but are not penetrating into the bilayers of $\mathrm{Bi}$, contrary to $\mathrm{Fe}$ and $\mathrm{Co}^{21}$

Before our magnetotransport results are presented, the effect of doping by the Tb adatoms shall be briefly discussed. The LEED and conductance experiments presented above suggest that the $\mathrm{Tb}$ atoms nucleate at defect sites of the pristine Bi(111) films. They thus do not act as additional scatters, but they can still act as dopants. The relative conductance in the final state is increased by about $0.7 \%$. Assuming that mobilities remain the same as for the clean surface, the charge carrier concentration of the $\mathrm{Bi}(111)$ surface states is increased by this amount, which is obviously provided by $\mathrm{Tb}$ excess coverage of $0.005 \mathrm{ML}$. From ARPES and previous transport studies a surface carrier concentration of $1 \times 10^{13} \mathrm{~cm}^{-2}$ has been deduced for the clean $\mathrm{Bi}(111)$ surface, ${ }^{7,8}$ i.e., a charge transfer of around 0.03 electrons is provided by each $\mathrm{Tb}$ adatom. As we will discuss in the next section, the charge transfer deduced from Hall measurements is found to be higher by factor of 2, but the estimate just made represents in fact a lower bound for the charge transfer, because any changes of the relaxation times and/or of the effective masses are not considered here.

\section{Magnetoconductance and Hall measurements}

Chemical shifts of atomic orbitals and charge transfer are the typical consequences of chemisorption. If $d$ electrons are involved in bonding, their effective magnetic moments are expected to be also strongly modified, as seen for $\mathrm{Fe}$ and $\mathrm{Co}$, e.g., Contrary to $\mathrm{Fe}$ and $\mathrm{Co}$, however, the high magnetic moment of $\mathrm{Tb}$ atoms $\left(10 \mu_{B}\right)$ is expected to be changed very little after adsorption since it is mainly due to $f$ electrons. We use here magnetotransport in order to identify the importance of spin-dependent scattering processes induced by $\mathrm{Tb}$ adsorption. ${ }^{14}$

Since $\mathrm{Tb}$ diffusion causes time-dependent changes of conductance, these cannot be neglected during magnetoconductance measurements. A sweep of the magnetic field from $0 \mathrm{~T}$ to $4 \mathrm{~T}$ takes around $1000 \mathrm{~s}$ in which the nucleation causes a $\Delta G_{\text {nucl }}$ of about $10-20 \mu \mathrm{S}$, which is of the same order of magnitude as $\Delta G(4 \mathrm{~T})$ induced by the classical magnetoeffect for a $20 \mathrm{BL}$ Bi film. ${ }^{7,14}$ Therefore a careful subtraction of $\Delta G_{\text {nucl }}$ is mandatory. By recording the change of $G$ over time at constant magnetic fields the effect of diffusion was estimated and the magnetoconductance signal correspondingly corrected. Exemplarily, the change of the conductivity versus time with both effects is shown in Fig. 4(a). The effect induced by diffusion can be deduced by fitting the curve before and after the sweep of the magnetic field. The carrier concentration during the magnetic sweep is changing and lower compared to initial value. Thus the conclusions drawn in the following represent a lower estimate. All measurements were carried out at the constant base temperature of $10 \mathrm{~K}$.

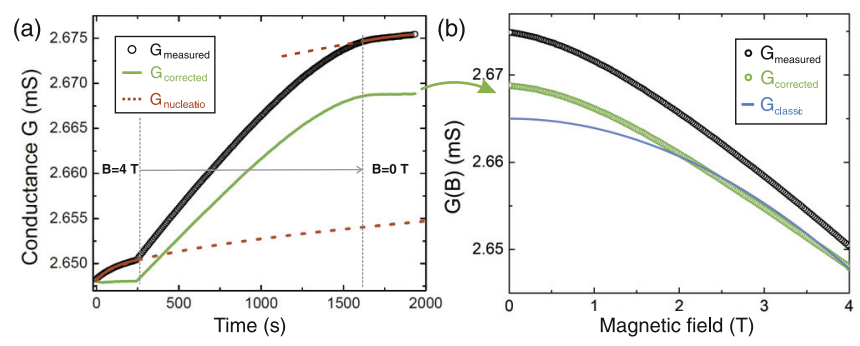

FIG. 4. (Color online) (a) Change of conductance vs time and magnetic field. The change of the magnetoconductance signal is superimposed by the effect of diffusion which can be estimated by fitting the values before and after the magnetic sweep. (b) The corrected magnetosignal as a function of the magnetic field. Same colors are used as in (a). Blue curve: classical magnetoconductance effect. 


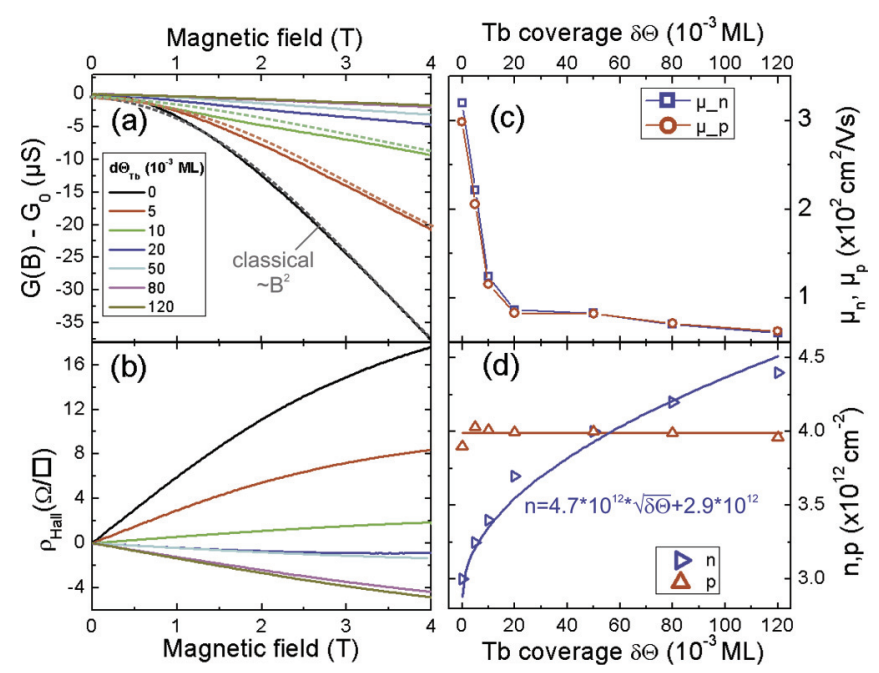

FIG. 5. (Color online) (a) Magnetoconductance measurements of $\mathrm{Tb} / \mathrm{Bi}(111)$ after subtraction of the contribution from $\mathrm{Tb}$ diffusion. (b) Hall resistivity data for the same set of measurements. The sign of the slope changes from positive to negative at concentrations above $1 \% \mathrm{ML} \mathrm{Tb}$. (c) The mobility decreases very strongly with excess Tb coverage. (d) The hole concentration is roughly constant whereas the electron concentration $\mathrm{n}$ is increasing upon $\mathrm{Tb}$ adsorption. The excess electron concentration follows in the low-coverage regime $\delta n \equiv n-n_{0} \approx 5 \times 10^{12} \mathrm{~cm}^{-2} \sqrt{\delta \Theta}$.

Figure 5 shows the corrected $G(B)$ (a) and $\rho_{\text {Hall }}(B)$ (b) data for different submonolayer coverages of $\mathrm{Tb}$ on a $20 \mathrm{BL}$ $\operatorname{Bi}(111)$ film at $T=10 \mathrm{~K}$. As demonstrated by comparing the black and the dashed black lines, which simulates the effect of classical magnetoconductance, the $G(B)$-curve shape of the clean Bi film is dominated this contribution. The adsorbed $\mathrm{Tb}$ atoms act as scattering centers, which, as qualitatively seen by the reduced curvatures of the curves, is a result of reduced carrier mobilities. Already, for smallest amounts of nominally 5\% ML, this effect is pronounced and demonstrates once again the sensitivity of magnetoconductance to adsorbates. The Hall resistivity $\rho_{\text {Hall }}$ has a positive slope for the clean film, in good agreement with our previous studies ${ }^{7}$ where we obtained values for the electron and hole concentration within the surface states of $n_{0} \approx 3 \times 10^{12} \mathrm{~cm}^{-2}$ and $p_{0} \approx$ $4 \times 10^{12} \mathrm{~cm}^{-2}$, respectively. Therefore the positive slope is due to the slightly higher hole concentration, although the hole mobility is lower compared to the electrons. Upon adsorption of $\mathrm{Tb}$ the slope of the Hall resistivity changes and reverses its sign at around $1-2 \% \mathrm{ML}$. For a detailed quantification of $\rho_{\text {Hall }}$ both carrier types need to be taken into account as well as their mobilities. The sign of the slope is determined by the balance between the ratio of their mobilities and concentrations and is positive (negative) if $\mu_{n} / \mu_{p}<(>) \sqrt{p / n} .^{14,22}$

In order to derive reasonable values for the carrier mobilities and concentrations the MC and Hall data have been fitted simultaneously, taking the effective masses from photoemission data. This procedure was the same as in our previous study ${ }^{14}$ where further details can be found. The results of the analysis of the magnetoconductance [see Fig. 5(a)] and Hall resistivity [see Fig. 5(b)] are shown in Figs. 5(c) and 5(d), respectively. The mobilities decrease quite strongly for
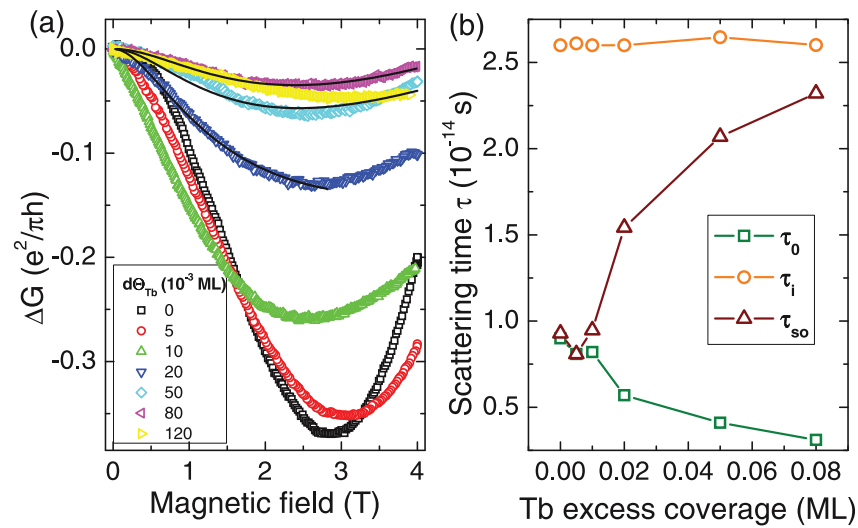

FIG. 6. (Color online) $G(B)$ data with the classical magnetoconductance contribution subtracted. All curves show WAL character. The amplitude decreases with $\delta \Theta_{\mathrm{Tb}}$. (b) Scattering time scales deduced from the data in (a) using Hikami theory. The elastic scattering time $\tau_{0}$ decreases, similar to the mobility. At the same time $\tau_{\text {so }}$ increases, so that a trend towards WL behavior results.

both carrier types from 300 to $150 \frac{\mathrm{cm}^{2}}{\mathrm{Vs}_{\mathrm{s}}}$ within a few percent of a ML adsorbate concentration and significantly stronger than in case of $\mathrm{Co}$ and $\mathrm{Fe}^{14}$ which might be caused by relaxation effects of the $\mathrm{Bi}$ lattice upon adsorption as discussed above. Simultaneously, there is a change in the carrier concentration. The electron concentration $n$ increases from 3 to $3.6 \times 10^{12} \mathrm{~cm}^{-2}$ after the adsorption of only $2 \%$ ML. A linear fit of the increase in this coverage window yields the amount of charge given to the Bi surface state by every individual $\mathrm{Tb}$ atom to be $\frac{\delta n}{\delta \Theta}=0.05 \frac{\mathrm{e}^{-}}{\text {Tb-atom }}$.

We now analyze the deviations from classical magnetoconductance [cf. with Fig. 5(a)]. These are attributed to weak localization (WL), which yields clear signatures of the spin-related scattering properties of $\mathrm{Tb}$ atoms. Figure 6(a) shows the corresponding data after careful subtraction of the classical magnetoconductance for different $\delta \Theta_{\mathrm{Tb}}$. In agreement with our former studies, the clean Bi films show WAL character, i.e., a decrease of conductance with increasing magnetic field at constant temperature. Starting with the curve for the clean Bi film, we see that this curve is governed by weak antilocalization, i.e., the defects on the clean Bi film cause strong spin dependent scattering, but spin remains a good quantum number, so that the $4 \pi$ symmetry of the spinor wave function governs the backscattering properties at the $\mathrm{Bi}$ surface. With increasing $\mathrm{Tb}$ concentration the minima of the curves in Fig. 5 become more and more shallow, indicating a gradual modification of this scenario.

For a quantitative analysis, the data have been evaluated by Hikami theory ${ }^{23}$ in order to determine characteristic scattering times for elastic, inelastic and spin-orbit scattering. For details of the analysis the reader is referred to Refs. 7 and 14. Best fits are represented by thin black lines, as exemplarily shown for some of the MR curves. The adjustment of the theory to the data was obtained by fixing first the inelastic scattering time at $\tau_{i}=2.5 \times 10^{-14} \mathrm{~s}$, justified by the fact that the temperature was set constant and no change of phonon scattering is expected. Iteratively it was then set free with the others fixed in order to get an optimal fit. Figure 6(b) shows the evaluation of all 
three relevant scattering times as a function of $\delta \Theta$. While the elastic time $\tau_{0}$ decreases together with the reduction of the carrier mobilities [see Fig. 5(c)], the spin-orbit scattering time $\tau_{\text {so }}$ increases significantly from 1 to $2.5 \times 10^{-14} \mathrm{~s}$ within the first $8 \%$ ML. The reduction of the spin-orbit scattering rate upon adsorption of adsorbates with nonvanishing magnetic moments is in agreement with recent findings for $\mathrm{Fe}$ and Co on $\mathrm{Bi}(111)$ films ${ }^{14}$ and reflects a WAL-WL transition. A similar observation has been made for the adsorption of $\mathrm{Fe}$ on $\mathrm{Bi}_{2-x} \mathrm{Cr}_{x} \mathrm{Se}_{3}$ films. ${ }^{24}$

Surprisingly, however, the effect of Tb adsorption is clearly weaker than for Fe and Co, while the trends are very similar; there is a reduction of the elastic scattering time, which correspondingly reduces the WAL effect. At the same time the overall shape of $\Delta G(B)$ changes, resulting in an increase of the spin orbit scattering time $\tau_{\text {so }}$. This increase is clearly much weaker than after adsorption of the same amount of $\mathrm{Fe}$ or $\mathrm{Co}$, typically by a factor of $2 .{ }^{14}$ Therefore there is no crossover into the $\mathrm{WL}$ regime by $\mathrm{Tb}$ adsorption for the coverage range up to $0.1 \mathrm{ML}$. The magnetoconductance remains still negative with respect to the zero-field value, but there are also differences. The mobilities of the electrons are more strongly reduced at the same excess concentrations of $\mathrm{Tb}$ than of $\mathrm{Fe}$ or Co. Furthermore, a fundamental difference between $\mathrm{Co}, \mathrm{Fe}$, and $\mathrm{Tb}$ is the charge transfer. While $p$ doping with 0.5 to 0.6 elementary charges per atom was found for former two elements, Tb behaves as a donor with a very small charge transfer. Finally, no indications of (surface) diffusion have been observed for $\mathrm{Co}$ and Fe. This correlates with the finding of low-temperature STM and of recently performed DFT calculations that an incorporation of these two elements on interstitial sites into the first bilayer takes place, ${ }^{21}$ whereas $\mathrm{Tb}$ atoms obviously stay on the surface.

As is well known, charge transfer by impurities depends on the type of bonding and on coordination. The different bonding character of $\mathrm{Co}$ and $\mathrm{Fe}$ on the one hand, and of $\mathrm{Tb}$ on the other, is reflected in their different adsorption sites. This is in qualitative agreement with the finding that $\mathrm{Fe}$ as adatom acts as donor, but as acceptor when it is bound in substitutional bulk defect sites on/in $\mathrm{Bi}_{2} \mathrm{Se}_{3} .{ }^{13}$ For the $\mathrm{Fe} / \mathrm{Bi}_{2} \mathrm{Se}_{3}$ system, it was shown further, that these different chemical environments result in different oxidation states. As a consequence the magnetic moment of Fe was found to be largest when incorporated in the bulk scenario where the charge transfer is smallest.

\section{E. Nucleation of Tb atoms: simulation}

The experiments strongly suggest that besides adsorption diffusion and charge transfer are further important processes. In order to support our conclusions from above (if only qualitatively), the process of adsorption and island formation in the initial stage has been modeled by means of nucleation theory. ${ }^{25,26}$ For the homoepitaxial Bi(111) system, it has been shown that the change of conductance upon adsorption can be indeed be accurately modeled. ${ }^{9,27}$ Here, we will extend this model including charge transfer (CT) and atomic steps of the $\mathrm{Bi}(111)$ films, which serve as perfect sinks for the diffusing monomers. The set of rate equations that reveal the main features of the $\Delta G / G_{0}$ curve (shown in Fig. 2) are

$$
\begin{aligned}
\frac{d N_{a}}{d t} & =f-k_{\text {step }} N_{a} N_{\text {step }}-2 k_{a} N_{a}^{2}-f\left(f t-N_{a}\right)-2 f N_{a}, \\
\frac{d N_{\text {step }}}{d t} & =-k_{\text {step }} N_{a} N_{\text {step }}, \quad \frac{d N_{\text {is }}}{d t}=k_{a} N_{a}^{2}+f N_{a} .
\end{aligned}
$$

The continuous generation of monomer adatoms $N_{a}$ is maintained by the incoming flux $\mathrm{f}\left(2 \times 10^{-5} \mathrm{ML} / \mathrm{s}\right)$ of atoms. Detachment processes from islands or defect sites are neglected as well as a coverage dependent sticking coefficient, which is assumed to be unity at our low substrate temperatures and low-coverage regime. The monomer density is reduced by diffusion of the monomers to steps or to domain boundaries as well as by formation of stable dimers with rates $k_{a}$ and $k_{\text {step }}$ (in $1 / \mathrm{s}$ ), respectively. The process of capturing of adatoms by already existing islands ( $-k_{\text {is }} N_{a} N_{\text {is }}$ ) turns out to be negligible in this low-coverage regime considered here, as revealed by explicit tests. Therefore we assume that the adatom density $N_{a}$ decreases either due to collision of two adatoms on the surface $\left(-2 k_{a} N_{a}^{2}\right)$ or by direct impact of an incoming atom onto an island $\left[f\left(f t-N_{a}\right)\right]$ or onto an adatom $\left(-2 f N_{a}\right)$. The second equation describes the decrease of nucleation sites $N_{\text {step }}$ at step sites and domain walls due to gradual adsorption of monomers. In particular, the diffusion of the adatoms to the former step sites is most crucial in order to explain the maximum observed in the conductance measurements. In our model, these defects trap the adatoms, i.e., detachment processes are neglected. Finally, the third equation describes how the island density increases by the collision of two adatoms $\left(k_{a} N_{a}^{2}\right)$ and the impingement of an atom from the atom beam on a Tb monomer $\left(f N_{a}\right)$. Larger islands than dimers are not considered.

The change of conductance was calculated by taking intrinsic defects and the actual surface defect concentration into account. The time-dependent (or, equivalently, coverage dependent for fixed fluxes) changes of the surface scatterers (monomers, (diatomic) islands, nucleation at steps) are determined by the rate equations given above. Assigning $\tau_{0}, \tau_{\mathrm{a}}$, and $\tau_{\text {is }}$ as the scattering times at intrinsic defects, adatoms and islands, respectively, the change of the conductance upon adsorption is given by $\Delta G_{\text {surf }} / G_{0}=-\tau_{0} /\left[\tau_{0}+\left(\tau_{a}^{-1}+\right.\right.$ $\left.\left.\tau_{\text {is }}^{-1}\right)^{-1}\right]^{-1}$. Please note that although the monomers diffuse to the steps, the scattering rates at the defect sites are assumed to remain the same as without trapped $\mathrm{Tb}$ atom and are already comprised in $\tau_{0}$. In general, the scattering time is proportional to the mean free path, i.e., inversely proportional to the defect density $\mathrm{N}$ and a corresponding probability $p\left[\tau \propto \ell \propto(N p)^{-1}\right]$, so that the relative change of conductance is expected to follow $\Delta G_{\text {surf }} / G_{0}=-\ell_{0}\left(p_{a} N_{a}+\right.$ $\left.p_{\text {is }} N_{\text {is }}\right) /\left[\ell_{0}\left(p_{a} N_{a}+p_{\text {is }} N_{\text {is }}\right)+1\right]^{-1}$ including the adatom density $N_{a}$ and the (diatomic) island density $N_{\text {is }} .{ }^{14}$ The scattering at the step-sites is determined by $\ell_{0}$, which is in our case around $14 \mathrm{~nm}$ (31 lattice sites) as, deduced from the SPA-LEED analysis above.

Apart from scattering at surface defects, the effect of charge transfer has to be considered. Assuming that relaxation times and effective masses remain constant upon the charge transfer from the $\mathrm{Tb}$ atoms into the surface states, the change of conductance by charge transfer is $G_{\mathrm{ct}} / G_{0}=\delta n / n_{0}$, where $\mathrm{n}_{0}$ and $\delta \mathrm{n}$ denote the pristine electron density within the $\operatorname{Bi}(111)$ surface states and the transferred charge, respectively (the 
(a)
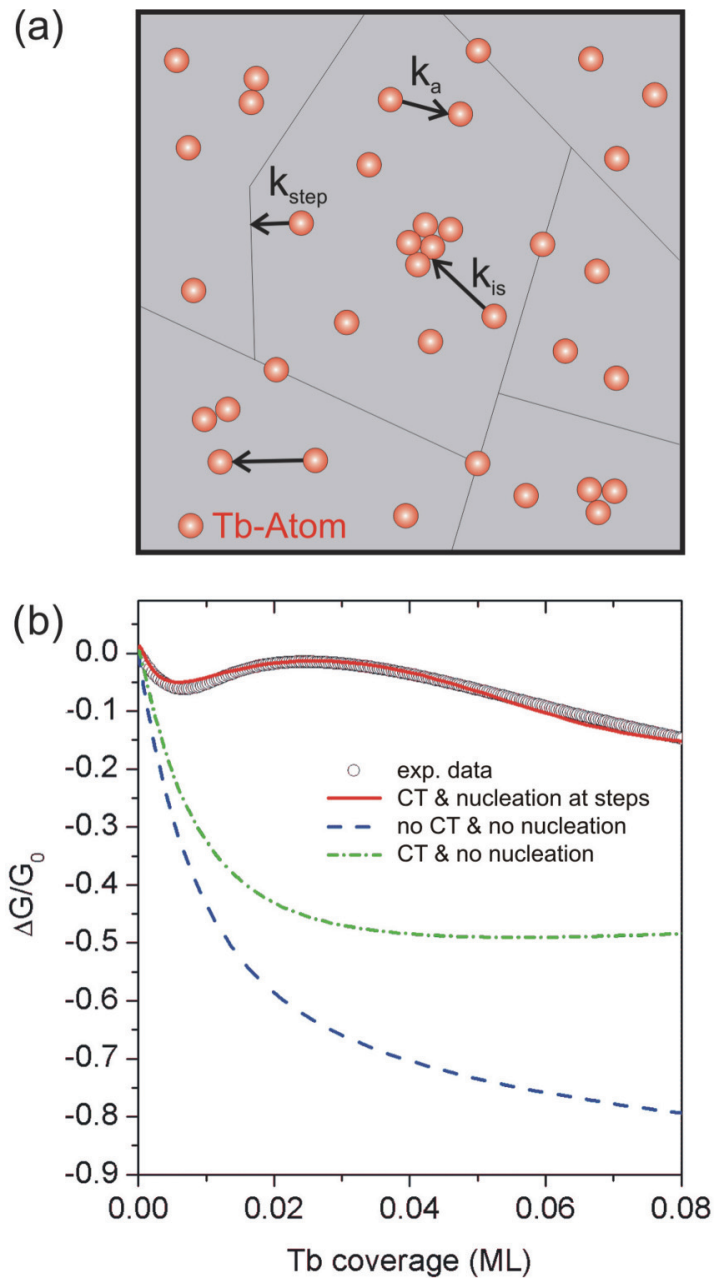

FIG. 7. (Color online) (a) Model of nucleation of Tb atoms on the surface of $\mathrm{Bi}$. The atoms either find other adatoms with a rate of $k_{a}$ or can be captured by the domain walls/steps (rate $k_{\text {step }}$ ) or by larger islands $k_{\text {is }}$. (b) Comparison between measurement (already shown in Fig. 1 at $T=10 \mathrm{~K}$ ) and simulation for three sets of parameters. Only if both nucleation of monomers at step sites and charge transfer (CT) are taken into account (red, solid curve), the experiment can be reasonably well described. For further details see text.

hole concentration stays almost constant and is not considered here). According to the discussion in context with Fig. 5(a), the change of the electron concentration can be parametrized by $\delta n \approx 5 \times 10^{12} \mathrm{~cm}^{-2} \sqrt{\delta \Theta}$, i.e., $G_{\mathrm{ct}} / G_{0} \approx \alpha \sqrt{\delta \Theta}$ with $\alpha \approx 1.7$.

The sum of both contributions $\left(\Delta G_{\text {surf }} / G_{0}+G_{\text {ct }} / G_{0}\right)$ is plotted in Fig. 7(b). The red (solid) line represent the best fit to the experimental data including both nucleation at step-sites and charge transfer. For comparison, the dashed curve shows the relative change of the conductance when neither charge transfer $(\alpha=0)$ nor nucleation $\left(k_{\text {step }}=0\right)$ is taken into account while for the dashed-dotted curve only charge transfer is considered. For the best fit, $\alpha$ is around 1.9 and only slightly larger as the straightforward estimation from above has revealed. Furthermore, for the capture rates, it was found that $k_{a} \ll k_{\text {step }}$, i.e., the nucleation at step sites is indeed a very likely process. As mentioned, processes of island decays, etc. are less important. Although this simple model cannot unravel all details of the diffusion and charge transfer process, it unambiguously shows that primarily diffusion is important and that the $\mathrm{Tb}$ captured by intrinsic defects of the $\mathrm{Bi}(111)$ films are immobilized.

\section{SUMMARY AND CONCLUSIONS}

In summary, we have shown that $\mathrm{Tb}$ adsorption on clean $\mathrm{Bi}(111)$ films is strongly influenced by diffusion and nucleation of $\mathrm{Tb}$ at defect sites such as steps on the Bi film even at temperatures as low as $10 \mathrm{~K}$. The peculiar adsorption of $\mathrm{Tb}$ on $\mathrm{Bi}(111)$ is confirmed by recent DFT studies. ${ }^{28}$

The peculiar $\mathrm{G}(\delta \Theta)$ dependency with the low-coverage minimum and maximum was successfully retraced by simulations, which supports our conclusions deduced from the experimental findings. Furthermore we find electron transfer of $\approx 0.05$ electrons per $\mathrm{Tb}$ atom to the $\mathrm{Bi}$ surface state in addition to a strong reduction of carrier mobilities.

Similar to our previous findings for $\mathrm{Fe}$ and $\mathrm{Co}$, also $\mathrm{Tb}$ turns out to be a scatterer with strong spin-orbit scattering of conduction electrons in the Bi surface states. Our starting point is always a $\mathrm{Bi}$ surface containing already intrinsic scattering centers that alter the spin orientation during the scattering process. Spin for these defects still seems to be a good quantum number, as seen by the clear WAL effect in magnetoconductance. Although the added concentrations of $\mathrm{Fe}, \mathrm{Co}$, and $\mathrm{Tb}$ are small, they make large contributions to electron scattering from the beginning, and dominate scattering already when a few percent of a monolayer are adsorbed as long as they are not condensed at the former defects. For Fe and Co, WAL turns into WL. This means that spin-orbit scattering is so strong by these impurities that all spin states are effectively mixed, i.e., spin is no good quantum number for these scattering processes and only weak localization is seen. The tendency is the same for adsorbed $\mathrm{Tb}$, but the scattering cross sections turn out to be smaller than for $\mathrm{Fe}$ and $\mathrm{Co}$, although $\mathrm{Tb}$ has a much higher magnetic moment than adsorbed $\mathrm{Co}$ and $\mathrm{Fe}$. This means that the high magnetic moment of the $f$ electrons plays no major role for scattering of the conduction electrons. The small scattering cross sections by the magnetic moment of the $f$ electrons may be due to their strong local confinement compared with the $s p$ electrons responsible for conduction, corresponding small overlap and the energetic separation between $f$ and $s p$ conduction electrons.

\section{ACKNOWLEDGMENT}

Financial support by the Deutsche Forschungsgemeinschaft (D.L.) and the DAAD (S.S.) is gratefully acknowledged. *tegenkamp@fkp.uni-hannover.de

${ }^{1}$ S. O. Valenzuela and M. Trinkham, Nat. Lett. 442, 176 (2006).

${ }^{2}$ S. Datta and B. Das, Appl. Phys. Lett. 56, 665 (1990).
${ }^{3} \mathrm{Ph}$. Hofmann, J. E. Gayone, G. Bihlmayer, Yu. M. Koroteev, and E. V. Chulkov, Phys. Rev. B 71, 195413 (2005). 
${ }^{4}$ J. W. Wells, J. H. Dil, F. Meier, J. Lobo-Checa, V. N. Petrov, J. Osterwalder, M. M. Ugeda, I. Fernandez-Torrente, J. I. Pascual, E. D. L. Rienks, M. F. Jensen, and P. Hofmann, Phys. Rev. Lett. 102, 096802 (2009).

${ }^{5}$ A. Stróżecka, A. Eiguren, and J. I. Pascual, Phys. Rev. Lett. 107, 186805 (2011).

${ }^{6}$ M. C. Cottin, C. A. Bobisch, J. Schaffert, G. Jnawali, A. Sonntag, G. Bihlmayer, and R. Möller, Appl. Phys. Lett. 98, 022108 (2011).

${ }^{7}$ D. Lükermann, S. Sologub, H. Pfnür, and C. Tegenkamp, Phys. Rev. B 83, 245425 (2011).

${ }^{8}$ T. Hirahara, I. Matsuda, S. Yamazaki, N. Miyata, S. Hasegawa, and T. Nagao, Appl. Phys. Lett. 91, 202106 (2007).

${ }^{9}$ G. Jnawali, C. Klein, Th. Wagner, H. Hattab, P. Zahl, D. P. Acharya, P. Sutter, A. Lorke, and M. Horn-von Hoegen, Phys. Rev. Lett. 108, 266804 (2012).

${ }^{10}$ N. Miyata, R. Hobara, H. Narita, T. Hirahara, S. Hasegawa, and I. Matsuda, Jpn. J. Appl. Phys. 50, 036602 (2011).

${ }^{11}$ Y. L. Chen et al., Science 329, 659 (2010).

${ }^{12}$ P. Roushan, J. Seo, C. V. Parker, Y. S. Hor, D. Hsieh, D. Qian, A. Richardella, M. Z. Hasan, R. J. Cava, and A. Yazdani, Nat. Lett. 466, 1106 (2009).

${ }^{13}$ T. Schlenk, M. Bianchi, M. Koleini, A. Eich, O. Pietzsch, T. O. Wehling, T. Frauenheim, A. Balatsky, J.-L. Mi, B. B. Iversen, J. Wiebe, A. A. Khajetoorians, Ph. Hofmann, and R. Wiesendanger, Phys. Rev. Lett. 110, 126804 (2013).

${ }^{14}$ D. Lükermann, S. Sologub, H. Pfnür, C. Klein, M. Horn-von Hoegen, and C. Tegenkamp, Phys. Rev. B 86, 195432 (2012).
${ }^{15}$ M. R. Scholz, J. Sánchez-Barriga, D. Marchenko, A. Varykhalov, A. Volykhov, L. V. Yashina, and O. Rader, Phys. Rev. Lett. 108, 256810 (2012)

${ }^{16}$ K. N. R. Taylor and M. I. Darby, Physics of Rare Earth Solids (Chapman and Hall, London, 1974).

${ }^{17}$ C. Tegenkamp, D. Lükermann, S. Akbari, M. Czubanowski, A. Schuster, and H. Pfnür, Phys. Rev. B 82, 205413 (2010).

${ }^{18}$ M. Kammler and M. Horn-von Hoegen, Surf. Sci. 576, 56 (2005).

${ }^{19}$ M. Horn von Hoegen, Z. Kristallogr. 214, 591 (1999).

${ }^{20}$ J. Wollschläger, E. Z. Luo, and M. Henzler, Phys. Rev. B 44, 13031 (1991).

${ }^{21}$ U. Gerstmann et al. (private communication).

${ }^{22}$ A. B. Pippard, Magnetoresistance in Metals (Cambridge University Press, Cambridge, UK, 1989).

${ }^{23}$ S. Hikami, A. I. Larkin, and Y. Nagaoka, Prog. Theor. Phys. 63, 707 (1980).

${ }^{24}$ M. Liu, J. Zhang, C. Z. Chang, Z. Zhang, X. Feng, K. Li, K. He, L. L. Wang, X. Chen, X. Dai, Z. Fang, Q. K. Xue, X. Ma, and Y. Wang, Phys. Rev. Lett. 108, 036805 (2012).

${ }^{25}$ J. A. Venables, G. D. T. Spiller, and M. Hanbücken, Rep. Prog. Phys. 47, 399 (1984).

${ }^{26}$ H. Brune, Surf. Sci. Rep. 31, 125 (1998).

${ }^{27}$ G. Jnawali, Th. Wagner, H. Hattab, R. Möller, A. Lorke, M. Horn-von Hoegen, e-J. Surf. Sci. Nanotech. 8, 27 (2010).

${ }^{28} \mathrm{U}$. Gerstmann et al. (private communication). In contrast to the adsorption of $\mathrm{Fe}$ and $\mathrm{Co}$, the energy of adsorption for $\mathrm{Tb}$ within the first bilayer was found to be $+0.7 \mathrm{eV}$ pointing towards a barrier-free outward relaxation. 